\title{
Um estudo do efeito de obstruções no escoamento de submeso na Camada Limite Convectiva
}

\author{
A study of the effect of obstructions the submeso flow in the Convective \\ Boundary Layer
}

\author{
Viviane da Silva Guerra ${ }^{1}$; Otávio Costa Avevedo ${ }^{2}$; Luiz Eduardo Medeiros ${ }^{3}$; Adriano Battisti ${ }^{4}$; \\ Thaís Freitas Dill ${ }^{4}$
}

${ }^{1}$ Aluna de Pós-Graduação em Física, Departamento de Física, Universidade Federal de Santa Maria, Santa Maria, Brasil;

2 Professor, Departamento de Física, Universidade Federal de Santa Maria, Santa Maria, Brasil;

${ }^{3}$ Professor, Departamento de Engenharia, Univesidade Federal do Pampa, Alegrete, Brasil;

${ }^{4}$ Aluno de Pós-Graduação em Meteorologia, Departamento de Física, Universidade Federal de Santa Maria, Santa Maria, Brasil;

${ }^{4}$ Aluna de Pós-Graduação em Meteorologia, Departamento de Física, Universidade Federal de Santa Maria, Santa Maria, Brasil

\begin{abstract}
Resumo
As equações construídas ao longo dos anos no estudo da Camada Limite Planetária, embora baseadas muitas vezes no âmbito experimental, foram criadas para situações idealizadas como, superfícies homogêneas horizontalmente, livre de obstáculos e condições de estado estacionário. No entanto tal situação de homogeneidade está longe da realidade, pois a superfície terrestre apresenta uma grande variabilidade quanto a topografia, cobertura de solo e áreas urbanas. O presente trabalho apresenta uma proposta para mostrar a influência da variabilidade da superfície local como o efeito de obstáculos no escoamento na escala de tempo ainda pouco conhecida, a submeso, na Camada Limite Convectiva. Para provar este efeito foi encontrado um Fator de Transmissão por setor do vento em oito estações micrometeorológicas presentes no sítio experimental de Pedras Altas na região do Pampa nos estado de Rio Grande do Sul. Tal Fator de Transmissão corrobora a influência local dos obstáculos na filtragem do escoamento possibilitando identificar em qual quadrante o vento está mais ou menos obstruído e ainda verificar em que escala de tempo a osbtrução exerce maior impacto.
\end{abstract}

Palavras-chave: submeso, Camada Limite Convectiva, Fator de Transmissão.

\begin{abstract}
The equations built over the years in the study of planetary boundary layer, although often based on experimental level, were created to idealized situations like, homogeneous surfaces flat, free from obstacles and steady state conditions. However such homogeneity situation is far from reality, because the earth's surface features a large variability in topography, ground cover and urban areas. This paper presents a proposal to show the influence of the variability of the local surface as the obstacle effect on the flow in the timescale still small known, the Submeso in Convective Boundary Layer. To prove this effect was found a Transmission Factor for each wind sector in the eight micrometeorological stations present in the experimental site of Pedras Altas in the Pampa region in the state of Rio Grande do Sul. Such Transmission Factor confirm the local influence of obstacles in filtering flow enabling identification of which quadrant the wind is more or less obstructed and even check in climbing time to osbtrução exerts greater impact.
\end{abstract}

Keywords: Submeso, Convective Boundary Layer, Transmistion Factor. 


\section{Introdução}

Processos não turbulentos na escala de submeso (Mahrt, 2009) são ainda pouco conhecidos, mas de relevante importância no que diz respeito a geração de turbulência na Camada Limite, segundo estudos recentes. O cisalhamento do vento, ainda que fraco, em condições estáveis provocados por movimentos não turbulentos com um grande número de Reynolds geram turbulência. O surgimento da turbulência pelo cisalhamento de submeso leva uma imprevisibilidade da turbulência, isso porque processos de submeso são ainda mal compreendidos e variam significativamente com as condições locais, como topografia, vegetação e mudam de local para local (Vikers e Mahrt, 2007).

Fluxos de submeso encontram-se em escalas maiores que a microescala mas bem menores que a mesoescala. e segundo Mahrt (2009) a escala temporal onde se encontram esses processos é pouco menos que alguns minutos.

O objetivo deste estudo é analisar a influência de obstruções na escala de movimentos de submeso na Camada Limite Convectiva (CLC) e comprovar a dependência local do submeso com tais obstruções. Para isso foram analisadas oito estações pertencentes ao Sítio de Pedras Altas-RS, região do pampa gaúcho, onde a Energia Cinética Turbulenta (ECT) horizontal presente em cada estação foi decomposta em várias outras escalas através da técnica da Decompoisição em Multiresolução (DM) (Howell e Mahrt, 1997). Tal técnica é uma ferramenta estatística poderosa, que separa a série temporal em várias escalas e é capaz de quantificar e apontar a frequência da ocorrência de um evento na escala de tempo.

Um ideia prévia para observação dos efeitos da obstrução do escoamento nas escalas de movimento foi feita utilizando uma técnica semelhante a usada por Fugita e Wakimoto (1982), através do cálculo de um Fator de Transmissão (FT). Através da DM é possível encontrar um FT para cada setor do vento em cada estação presente no sítio experimental possibilitando identificar a escala de tempo mais afetada pela obstrução.Contudo a ideia desse trabalho não é encontrar as causas do surgimento de movimentos de submeso e sim comprovar sua existência na CLC, sua colaboração na turbulência através de sua dependência local.

\section{Materiais e Métodos}

O sítio experimental de Pedras Altas é uma região de Pampa Gaúcho localizado a uma latitude de $31^{\circ} 44^{\prime} \mathrm{S}$ e longitude $53^{\circ} 35^{\prime} \mathrm{W}$ com uma altitude em torno de 373 metros (Figura 1) e ocupa uma área em torno de dois mil metros quadrados com uma vegetação local composta basicamente por gramíneas e árvores de pequeno e médio porte, como a direita da Figura 1 a presença de uma extensa plantação de Acácias que influenciará nos resultados desse trabalho. As estações micrometeorológicas mantiveram-se em funcionamento em torno de um mês, de agosto a setembro de dois mil e treze. Os dados utilizados para o cálculo da ECT horizontal foram velocidade e direção do vento, zonal e meridional. Para a análise foram separados vinte e três dias, (devido a problemas nos dados dos outros dias) das oito horas e cinquenta e quatro minutos da manhã às dezessete horas e vinte quatro minutos da tarde. As oito estações foram dispostas a uma pequena distância de separação umas da outras, em torno de $1 \mathrm{~km}$, para verificar que mesmo a uma pequena distância a ECT pode sofrer a influência de diferentes setores do vento devido a presença de obstáculos. A orografia local e algumas árvores próximas em algumas estações filtram significativamente a velocidade do escoamento. As torres possuíam diferentes frequências de registros, as de baixa frequência, com uma frequência de dois minutos e as de alta 10 hertz.

A influência local sobre a ECT horizontal será provada fazendo uma análise no seu comportamento espectral da ECT na camada convectiva utilizando a técnica da Decomposição em Multiresolução (Howell e Mahrt, 1997). Através desta técnica é possível identificar que o espectro gerado segue uma lei de escala e é totalmente vulnerável a possíveis obstruções no escoamento. A DM é uma ferramenta estatística avançada e indicada para estudos de séries temporais quando se quer quantificar um evento e a frequência da sua ocorrência em uma determinada escala.A DM decompõe o sinal em suas escalas, onde a escala de tempo do pico do espectro define que escala temporal a maior parte das flutuações ocorre.

O espectro e o coespectro gerado pela multirresolução é interpretado em termos das médias móveis não ponderadas no método da transformada de Haar através de ondeletas descritas por Mallat (1989). Vikers e Mahrt (2003) descrevem rapidamente como o processo da Decomposição ocorre.

A ECT total associada a todas as escalas do movimento é dada pela soma da curva do espectro de energia cinética, desde escalas dos pequenos turbilhões a escalas dos maiores turbilhões. Assim a ECT total associada ao fluxo turbulento é dada pela integral da densidade espectral S(k) (Equação (1)) variando nos limites de escala de zero a infinito que equivale a variância total do evento.

$$
E=\int_{0}^{\infty} S(k) d k=\sigma_{k}^{2}
$$

Para o cálculo da obstrução do escoamento foi utlilizada a ideia semelante a proposta de Fujita e Wakimoto, (1982), Eles apresentaram uma proposta para correção 
do vento obstruído apresentando uma maneira de calcular o Fator de Transmissão como mostra a Equação (2).

$$
\psi_{i}=\frac{v}{v_{i}}
$$

onde $\psi_{i}$ é o fator de transmissão, $v$ é a velocidade de vento obstruído, e $v_{i}$ é o vento livre variando em $i$ estações micrometeorológicas.

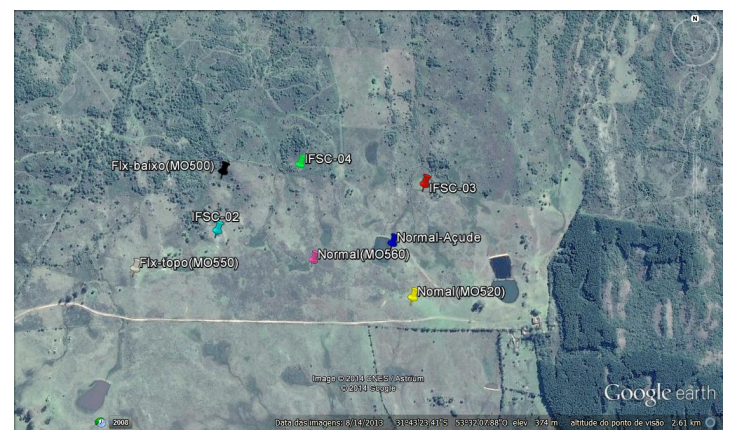

Figura 1: Sítio experimental de Pedras Altas-RS e a distribuição das oito estações micrometeorológicas

\section{Resultados e Discussões}

O espectro da ECT média horizontal neste trabalho é obtido pela média das somas das partes turbulentas entre $u^{\prime}$ e $v^{\prime}$. Esse resultado aponta um tratamento estatístico bastante importante para qualificar a turbulência na CLA, pois fornece a soma das variâncias em cada componente do escoamento.

$$
\frac{E C T}{m}=0,5\left(\overline{u^{\prime 2}}+\overline{v^{\prime 2}}\right)=\overline{E C T},
$$

A ECT média horizontal para movimentos no alcance do submeso seguiu uma lei de escala para ambas as estações (Figura 2), aumentando exponencialmente para escalas de tempo de menor frequência, o que está de acordo semelhantemente com resultados encontrados por Mahrt (2009) e Acevedo et al. (2014). A ressalva está na divisão da escala entre subesmo e a turbulência encontrada por esses autores, em torno de um a dois minutos e a encontrada neste trabalho, de cerca de 40 minutos. Uma possível explicação porque isso ocorre, é que nos trabalhos anteriores foram analisados dados noturnos, em que as escalas temporais do movimento turbulento são reduzidas, permitindo a ocorrência de submeso em menores escalas de tempo.

Como resultado preliminar, a ECT média horizontal em movimentos de submeso está concentrada nas baixas frequências o que já era de se esperar. Este padrão é visto em todas as estações, porém ainda é possível perceber uma diferença na quantidade de energia de estação para estação, como mostra a Figura 2 por exemplo, a Flx-baixo(MO500) em relação a IFSC-03. A IFSC-03 é a estação que possui maior quantidade de ECT associada a escala de menor frequência, enquanto que a Flxbaixo(MO500) é a que apresenta menor ECT.

Essas diferenças encontradas motivaram o objetivo deste trabalho, que é encontrar o que está provocando tais diferenças. Acredita-se que as diferenças encontradas no espectro da ECT média horizontal no âmbito do submeso em cada estação, seja devido a diferentes tipos de obstruções existentes, como orografia e cobertura do solo.

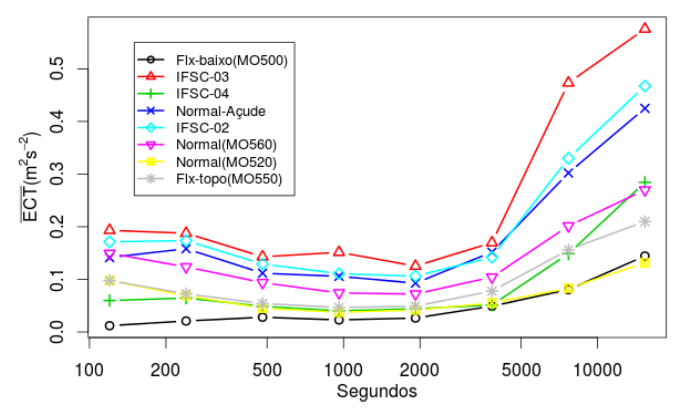

Figura 2: Espectro da Energia Cinética Turbulenta média horizontal total

Para encontrar a influência local em cada estação foi feito o cálculo da obstrução local em cada estação para cada setor do vento (Aqui será mostrada somente quatro estações que mais apresentaram diferenças). Os dias foram classificados por quadrante sendo que os dias que apresentaram muita flutuabilidade quanto a direção foram retirados. Após a separação dos dias por setor foi calculada então a ECT média horizontal para cada setor como mostra a Figura 3.

Diferenças significativas de estação para estação foram encontradas, mas principalmente entre os diferentes setores, o que sugere a dependência local de movimento de submeso e que este é afetado pelos diferentes tipos de obstruções existentes. Nos dias de vento de setor Nordeste (Figura 3 a)por exemplo a energia associada ao submeso é maior para a estação IFSC-03 e Normal-Açude, Porém quando o vento estava de Sudeste (Figura $3 b$ ) a estação com maior energia na baixa frequência é a $\mathrm{MO}(560)$, o mesmo acontece em outros setores (Figura 3 $c$ e d). Tais resultados indicam a influência de obstáculos e obstruções em determinados setores do vento. Em algumas estações como a Flx-baixo(MO500) a energia é muito pequena em todas as escalas, mostrando que nesta estação as obstruções estão filtrando o vento tanto 
em baixa quanto em alta frequência. $\mathrm{O}$ mesmo ocorre para as estações Flx-topo(MO550) em ambos os setores.
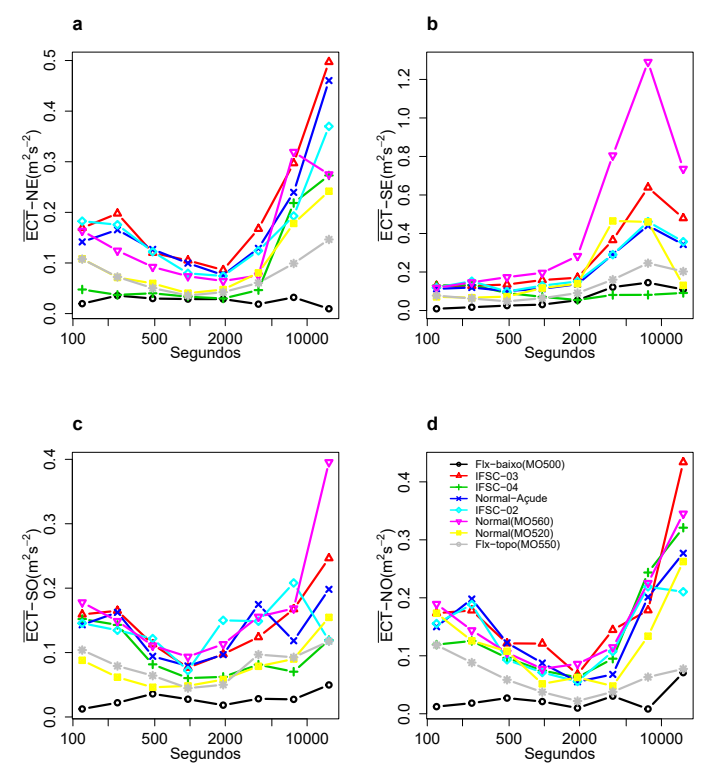

Figura 3: Energia Cinética Turbulenta média horizontal por setor da direção do vento. Em a pelo setor Nordeste (NE) e, b pelo setor Sudeste(SE), em c pelo setor Sudoeste(SO), e em d pelo setor Nordeste(NO)

Estes resultados motivaram a questão fundamental a ser investigada neste trabalho, que é identificar as escalas de tempo em que as diferentes obstruções filtram a energia do escoamento. Ou seja verificar um Fator de Transmissão que identifique em qual escala de cada setor a obstrução é mais preponderante. Para encontrar um FT para cada setor e escala temporal foi encontrada a estação que apresenta maior ECT. Assume-se que a estação que apresentar maior energia em determinado setor e escala temporal está desobstruída naquelas condições.

O FT, que quantifica o efeito das obstruções, é calculado como a razão entre o espectro de ECT em um dado setor e escala do tempo pelo valor desobstruído (máximo observado no sítio) daquele setor e escala temporal. Quanto mais próximo de 1 for esta razão mais desobstruído será o setor, enquanto valores próximos a zero indicam grande obstrução.

Nas estações IFSC-03 e a Normal(MO520) é interssante notar que os resultados indicam obstruções principalmente no setor Sudeste e para as escalas maiores de tempo, isso sugere que possivelmente esse setor está sendo influenciado pela presença de uma plantação de Acácias a direita das estações IFSC-03 e Normal(MO520)contribuindo para um FT menor nas baixas frequências(Figura $4 a$ e $c$ ). Diferentemente a estação Normal(MO560)(Figura $4 b$ ) apresenta um FT mais alto da rede de estações próximo de 1 para o setor SE, sugerindo que quando o vento está desse setor, nessa estação o vento encontra-se praticamente desobstruído, mas variavelmente nas diferentes,obstruído no setor NE (Figura 4 b) com significativa desobstrução na escala de submeso. Já na Figura $4 d$ nota-se que o escoamento está sendo filtrado apenas nas baixa frequências, de modo que o FT decresce esponencialmente com a escala de tempo.
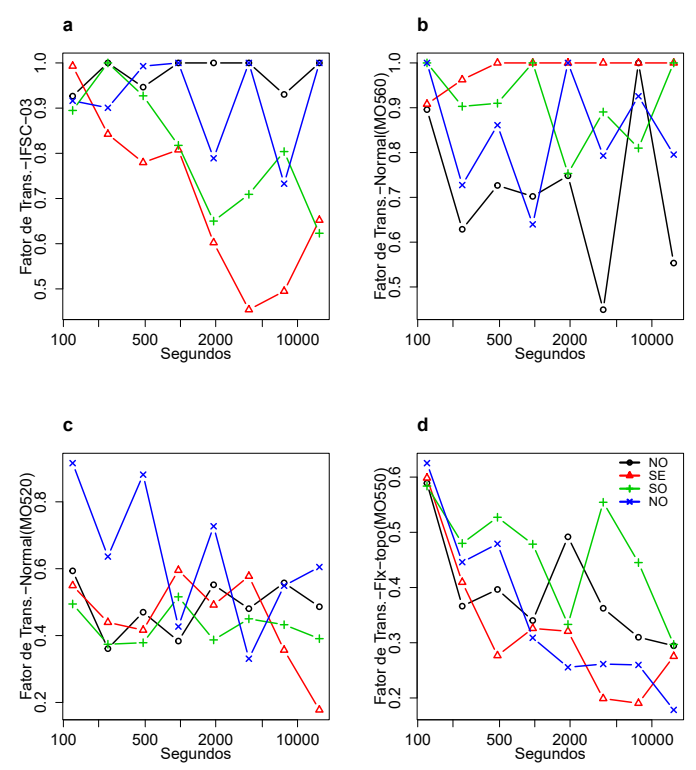

Figura 4: Fator de Transmissão por setor do vento. Em preto pelo setor Nordeste, em vermelho pelo setor Sudeste, em verde pelo setor Sudoeste e azul pelo setor Noroeste

\section{Conclusões}

No presente trabalho foi apresentado um esforço inicial para compreender o quanto o efeito de obstrução afeta o escoamento em diferentes escalas temporais. Isto é um esforço pioneiro, que pode auxiliar na compreensão dos processos que controlam o escoamentode submeso, e como características superficiais como a topografia ou proximidade a obstáculos de diferentes dimensões afetam a energia do escoamento.

Alguns resultados apresentados são bastante promissores. É possível notar que algumas estações apresentam reduções predominantemente em pequenas escalas temporais do escoamento, típicas de turbulência, sendo menos afetadas em escalas de Submeso. Em outros casos, são as escalas mais longas, de Submeso as mais reduzidas. Apareceram indícios de que padrões de topografia têm influência, bem como um possível efeito causado pela plantações de árvores a sudeste da rede de estações. De qualquer forma, em muitos casos não foi possível associar uma causa física a padrões observados e é possível que isso seja causado pela análise, ainda 


\section{Referências}

Acevedo, O. C., Costa, F. D., Oliveira, P. E. S., Puhales, F. S., Degrazia, G. A., Roberti, D. R. (2014). The influence of submeso processo on the stable boundary layer similarity relationships. Journal of the Atmospheric Sciences, 71, 207-225.

Howell, J. F., Mahrt, L. (1997). Multiresolution flux decomposition. Boundary-Layer Meteorology, 81, 117-137.

Mahrt, L. (2009). Characteristics of submeso winds in the stable boundary layer. Boundary-Layer Meteorol, 130, $1-14$.

Mallat, S. (1989). Multiresolution flux decomposition. IEEE Trans Pattern Anal Machine Intell, 11(7), 674-693.

Vikers, D., Mahrt, L. (2003). The cospectral gap and turbulent flux calculations. journal Atmospheric and oceanic technology, 20, 660-672.

Vikers, D., Mahrt, L. (2007). Observations of the crosswind velocity variance in the stable boundary layer. Journal of Boundary-Layer Meteorol, 7, 55-71. 